\title{
Retos de la competitividad urbana en México
}

\section{Challenges of urban competitiveness in México}

\author{
Cabrero, Enrique (2013), Retos de la competitiVidad urbana, \\ CIDE, MÉXICO, 372 PP., ISBN: 978-6-07-784-352-8.
}

Muchos son los desafíos que día con día enfrentan las ciudades al intentar aprovisionar todos aquellos satisfactores que permiten la reproducción social en su concepción más amplia; en la actualidad se gestan diversos cuestionamientos en torno a la capacidad de estos conglomerados urbanos frente al fenómeno de la globalización y su forma de inserción, así como su participación, en donde las particularidades podrían reflejar una diferencia en términos de competitividad.

En el libro que a continuación se describe, el autor provee un panorama general del acontecer de las ciudades mexicanas inscritas en un marco de competitividad, lo cual no es el fin último del texto, sino que forma parte de un análisis cuya pretensión es sentar las bases para la toma de decisiones al momento de diseñar políticas públicas que permitan a las ciudades forjar un desarrollo y bienestar en términos de las necesidades de la colectividad.

La obra está compuesta por cuatro capítulos en los que se abordan aquellos elementos que hacen de las ciudades espacios no sólo para la producción de bienes y servicios, sino lugares generadores de conocimiento, creación de nuevas ideas y definición de nuevas formas de relación social. En palabras del autor "es en las ciudades donde surgen las nuevas formas de ciudadanía, de gobierno, de convivencia, de movilización y de realización humana” (p. 15), por lo que es fundamental entender su proceso de conformación debido a que marcan el diario vivir o, en algunos casos, el subsistir, así como el ritmo de crecimiento, bienestar y progreso. Su análisis, por tanto, no es exclusivo de una disciplina en particular, sino que incorpora diferentes perspectivas para su mejor entendimiento, porque en las ciudades es donde habita más de $50 \%$ de la población en el mundo y, en el caso de México, más de tres cuartas partes de la población.

También se retoman las concepciones en torno a la significancia del término competitividad y su repercusión en el contexto nacional; así como su expresión más allá de las fronteras y cómo este concepto ha sido paulatinamente trastocado y revalorizado. Sin embargo, en palabras del 
autor, la competitividad reviste una serie de procesos que en su conjunto permite la focalización de un proyecto integral de ciudad; para ello la entidad deberá ofrecer los elementos mínimos indispensables para la gestación de las actividades económicas y sociales, así como la afluencia de conocimiento, polos de innovación tecnológica, existencia de empresas innovadoras e infraestructura y equipamiento urbano que faciliten dichas actividades, así como capital social que fortalezca la cooperación entre grupos empresariales, sociales y de ciudadanos, así como capital humano altamente capacitado y una dinámica de sustentabilidad regional que preserve los recursos naturales y no deteriore indiscriminadamente el medio ambiente.

No obstante, se hace hincapié en que la existencia de estos elementos no es suficiente, porque es preciso un sistema de gobernanza que haga posible la conexión de todos los elementos en un circulo virtuoso; es decir, la existencia de un marco institucional y regulatorio propicio y ágil, así como estructuras gubernamentales de calidad, construcción de redes de políticas públicas para el desarrollo urbano integral y, derivado de todo esto, una acción pública de alta intensidad, lo que significa capacidad de generar consensos y acuerdos entre grupos diversos de la sociedad y gubernamentales.

En este contexto, se reflexiona a través de diversos cuestionamientos en torno a la condición y capacidad que guardan las ciudades mexicanas para generar un ambiente propicio para la competitividad ¿cómo saber si una ciudad en particular posee estos atributos? ¿Cómo entender en qué medida los posee? ¿Cómo poder comparar unas ciudades con otras en este perfil de atributos para la competitividad? ¿Cuáles son los que poseen las ciudades mexicanas? ¿En qué medida se puede hablar de ciudades competitivas en México? ¿Cómo compararlas y observar cada una respecto a su evolución en el tiempo?

En el primer capítulo se realiza una actualización del índice de competitividad que se generó en el 2003, este es el primer acercamiento a la generación de datos que permiten hacer inferencias del comportamiento y características de las ciudades a manera de indicadores, y reviste una atención especial porque se propone una metodología que no pretende ser exhaustiva a la hora de identificar todas las variables, sino más bien definir aquéllas realmente significativas en un ejercicio también segregativo, en función de la información disponible; para ello se hace una revisión y uso de métodos cuantitativos y cualitativos, donde se procura que se refleje fehacientemente información que permita la realización de comparativos en primera instancia, así como la generación de procesos de aprendizaje sobre experiencias diversas y sobre la propia evolución de la ciudad a través del tiempo. 
Para el cálculo del índice de competitividad el autor se basa en el método de análisis factorial que ha sido utilizado en diversos estudios reconocidos en otros países, entre los cuales se encuentra el "The global competitiveness report”, publicado por The World Economic Forum, el cual, de acuerdo a sus conjeturas, es un método que permite aislar diversas dimensiones dentro de un grupo de variables relacionadas entre sí; es decir, separa patrones comunes de variación que facilitan el análisis de alguna situación en particular e identifica dónde se generan, así como la relación de las variables en su interior.

El estudio se focalizó en cuatro subíndices denominados componentes: 1) económico, 2) institucional, 3) socio-demográfico y 4) urbano-ambiental; los cuales consideran que responden a la definición de competitividad urbana que busca explicar el índice.

Con lo anterior, se permite la observación de la situación de competitividad de sesenta ciudades mexicanas en los años 2003, 2007 y 2011, donde también se refleja su evolución a través del tiempo. Por otra parte, se llevó a cabo el cálculo del índice de competitividad ampliado, el cual integra más variables que los índices anteriores debido a la disponibilidad de nuevas bases de datos que el INEGI ha generado en los ámbitos de seguridad pública a nivel municipal, nivel de profesionalización de funcionarios municipales, marco normativo reglamentario municipal y políticas específicas en manejo de desechos, control interno, transparencia y algunas otras. Por ello, el análisis inferido provee mayores elementos de precisión que permiten comprender de mejor manera la situación de competitividad de las ciudades.

El libro menciona que 14 ciudades se mantuvieron a lo largo de los tres años de referencia (2003, 2007 y 2011) en los primeros 20 lugares de competitividad urbana del país, lo cual nos muestra, sin duda, que se trata de los motores urbanos de la competitividad y que son un referente obligado en términos de sus prácticas a nivel nacional; sin embargo, si bien son líderes nacionales, están muy rezagadas en comparación con otras ciudades del mundo.

Según estudios recientes la ciudad de México ocupa el lugar 74; Chihuahua, el 106; Monterrey, 143; y Guadalajara, 180, entre quinientas ciudades analizadas. En este sentido, la generación del índice tiene la intención de que aquéllos que toman decisiones en los tres órdenes de gobierno cuenten con un mayor conocimiento sobre la situación de sus ciudades y, de esta manera, puedan llevar a cabo actividades encaminadas a crear las mejores condiciones posibles para promover la competitividad.

Por lo tanto, el diseńo de políticas públicas deberá incluir elementos transversales que logren un cambio tangible en el corto plazo en las ciudades mexicanas, estas políticas sentarán las bases para el mejor aprove- 
chamiento de sus recursos tanto en la esfera económica, la esfera política, la social, o el marco de la sociedad civil. No obstante, también se advierte que si las ciudades estudiadas no logran mejorar de manera acelerada en los próximos ańos, muy probablemente los rezagos nacionales con otros países se agudizarán.

En el segundo capítulo se intenta desmitificar ciertas prácticas que en la actualidad todavía se presentan respecto a lo tradicionalmente concebido como más competitivo en una región o localidad en el caso de México, en las que se ofrece mano de obra a una tasa salarial baja, recursos naturales baratos y abundantes, y regulaciones gubernamentales blandas con la esperanza de captar la mayor inversión disponible a un costo aparentemente desconocido; pero una ciudad con niveles salariales bajos y con índices de alta desigualdad y exclusión social, no necesariamente atrae el capital humano especializado y de altas capacidades, el cual es un componente crucial para avanzar en el perfil de competitividad de una ciudad.

Tampoco, por cierto, el uso indiscriminado de recursos naturales y el consecuente deterioro ambiental, o la ausencia de un sistema de regulación claro y estable, atraen la inversión de alto valor agregado de empresas generadoras de redes de inversión y empleo, así como conexiones de flujos financieros y tecnológicos a nivel mundial. Por lo que se integra un estudio sobre el perfil y liderazgo regional de ciudades.

Más allá de los niveles de competitividad de las ciudades y de sus perspectivas para tener presencia internacional y formar parte de las redes de la economía global, es necesario tener claro el papel que las diversas ciudades del país juegan en sus entornos y qué tan determinantes son en las economías regionales del territorio nacional.

Se presentan otros ángulos del sistema urbano nacional al agrupar ciudades con un perfil moderno, otras con un perfil de transición, y otras con uno tradicional, además se destaca el liderazgo regional de las mismas.

Queda claro en este análisis que hay vocaciones específicas que deben ser potenciadas de una manera más decidida. Además, es posible identificar clústeres más amplios de conjuntos de ciudades que deberán constituir una estrategia explícita de promoción desde otros niveles de gobierno, con vías a consolidar un sistema de ciudades más competitivo y funcional en el país.

En el tercer capítulo se presenta una discusión amplia sobre competitividad y cohesión social. El autor plantea que el conjunto de ideas y valores a los que da lugar el concepto de cohesión social resulta indisociable de la competitividad y el desarrollo económico. Se acepta que en cualquier ciudad existe una parte de la población en situación de exclusión y pobreza; lo que se propone es entender los diferenciales que muestran 
una mayor o menor gravedad del problema de la pobreza urbana en las diversas ciudades de México; para ello, se realiza un análisis comparado de las ciudades mexicanas identificando los diferenciales en variables relacionadas a procesos de exclusión social y relacionando estos al nivel de competitividad que las ciudades presentan.

Los resultados del estudio son claros, en la medida que las condiciones de exclusión educativa en grupos marginados se hacen más severas, la competitividad urbana tiende a disminuir; por otra parte, la falta de acceso a la seguridad social de estos grupos marginados sin duda genera, como dicen los autores, una cadena de exclusiones que va del trabajo al conjunto de los derechos sociales, lo cual también muestra una relación con baja competitividad de las ciudades.

Finalmente, y a partir de estas observaciones, se recalca que cuando de competitividad se trata es necesario considerar no sólo las variables estrictamente económicas, sino también los procesos de reacomodo social y la capacidad de absorción de los migrantes a la ciudad para incorporarlos a la actividad económica de la misma. El actual es un modelo de desarrollo altamente dependiente del capital humano y éste no se logra atraer o retener en una ciudad donde predomina un ambiente socialmente degradado.

Por último, en el capítulo cuatro, se da cuenta del componente cualitativo de esta investigación al presentar los resultados de una serie de seminarios-taller que se organizaron con la participación de más de una centena de funcionarios municipales responsables de la agenda del desarrollo y la competitividad de municipios urbanos del país. Para ello se convocó a autoridades de ciudades del centro del país, de la zona fronteriza del norte, de la región sur, de ciudades estrechamente vinculadas a la actividad petrolera, de ciudades vinculadas a la actividad turística, así como al conjunto de funcionarios de municipios y delegaciones de las tres zonas metropolitanas más grandes del país: Guadalajara, Monterrey y la ciudad de México.

En estos seminarios se presentaron los resultados del índice y se procedió a una amplia discusión sobre el método utilizado; se debatió qué tanto los resultados, a su entender, reflejan la situación de la ciudad; se habló sobre los factores de la política pública de promoción de la competitividad que se complican debido a las debilidades institucionales de los gobiernos municipales; así como a dificultades políticas y administrativas para impulsar la coordinación metropolitana y la participación de grupos empresariales y sociedad civil.

A manera de conclusión, se esgrime que para que una ciudad logre altos niveles de competitividad debe ser capaz, entre otros factores, de atraer talentos (capital humano) y empresas con un espíritu innovador: 
conglomerados urbanos que se insertan en la dinámica de una sociedad y una economía del conocimiento, un escenario en donde la creatividad, las ideas, el conocimiento científico, el desarrollo tecnológico y la innovación, marcan el perfil dominante de la ciudad.

Muestra de esto son las buenas prácticas en materia de desarrollo urbano, crecimiento y bienestar que corresponden a países que promovieron un conjunto de motores para la competitividad; es decir, que lograron conformar un grupo de ciudades con altos estándares de vinculación entre sus empresas (sector productivo), sus grupos de científicos y tecnológicos (sector académico) y sus gobernantes y funcionarios locales (gobierno).

Conforme al texto, en México la vinculación entre estos tres sectores: la empresa, la universidad y el gobierno local todavía es muy escasa o incluso inexistente. El problema de esta realidad es que aun cuando se reúnan muchas de las características de una sociedad competitiva, sin el componente de una vinculación ágil entre los sectores ya mencionados, es probable que la ciudad no logre dar el salto determinante hacia la economía del conocimiento. La promoción de la vinculación entre el sector productivo, académico y gubernamental, para generar innovación y competitividad, debe ser parte indispensable de las políticas públicas.

Definitivamente, hace falta mucho que hacer en el caso de las ciudades mexicanas, difícilmente en el corto plazo se podrán reflejar frutos que verdaderamente sean el resultado de una acción conjunta; evidentemente el trabajo realizado y plasmado a lo largo del texto y de las diversas conjeturas que se han visualizado son tema de reflexión.

Las medidas que se proponen podrían en determinado momento direccionar de manera positiva y promover un cambio gradual en el alcance y ofrecimiento de mejores condiciones de vida; si bien es cierto que existen ciudades líderes en el tema de competitividad, también lo es que hay un trecho bastante significativo que las separa de las demás; es imprescindible redefinir un modelo de ciudades para que, efectivamente, responda a las necesidades actuales y no se quede en un ejercicio aislado.

El libro también señala la importancia de la municipalidad, porque es en el nivel local donde se consolidan y expresan las relaciones económicas, sociales, políticas, urbano-ambientales; es preciso pensar en modelos que permitan la permanencia de proyectos de largo alcance, que muchas veces se ven truncados por los periodos de gobierno; asimismo, es necesaria una articulación hacia los niveles de gobierno superiores y la búsqueda de la acción conjunta y ampliamente consensuada. Es preciso permear en las estructuras la urgencia de conducir el desarrollo con una visión coordinada y sustentada en la corresponsabilidad, dejando de lado intereses parciales y sacando mejor partido de las potencialidades que cada municipio, estado o región contiene. En palabras del autor "las ciudades del 
mundo se despiertan, se agilizan y buscan posicionarse mejor, sería lamentable que en México no lográramos ese despertar y nuevamente perdamos otra oportunidad para avanzar en el desarrollo y el bienestar social” (p. 36).

El texto, sin lugar a dudas, refleja la importancia y necesidad de la transición hacia la competitividad en nuestras ciudades, ofrece un momento de reflexión respecto al estado que guardan, así como directrices que podrían ayudar a quienes toman decisiones para diseñar mecanismos que reivindiquen su camino en una esfera de verdadera conciencia dirigida hacia el desarrollo continuo y la sustentabilidad.

Recibida: 30 de junio de 2014. Aceptada: 3 de julio de 2014.

Oscar Montaño-Arango Universidad Autónoma del Estado de Hidalgo Correo-e: omontano@uaeh.edu.mx

Oscar Montaño-Arango. Mexicano. Es doctor en ingeniería (sistemas de planeación) por la Universidad Nacional Autónoma de México, labora en el área académica de Ingeniería del Instituto de Ciencias Básicas e Ingeniería perteneciente a la Universidad Autónoma del Estado de Hidalgo. Pertenece al Sistema Nacional de Investigadores, nivel I. Sus líneas de investigación son el análisis de sistemas y la planeación. Entre sus publicaciones sobresalen "Medición de la innovación en las pymes mediante la aplicación de métodos multicriterio", Mercados y Negocios, 14 (2), Universidad de Guadalajara, Guadalajara, pp. 169-186 (2013), "Modelo sistémico para la conformación de un clúster turístico regional de naturaleza sustentable", Economía, Sociedad y Territorio, XII (39), El Colegio Mexiquense, A. C., Zinacantepec, pp. 493-525 (2012), "Knowledge, learning and development: the challenge of small and medium enterprises to global competition", en Piotr Pachura (ed.) The economic geography of globalization, Intech, Croacia, pp. 99-112 (2011); "Modelo que identifica la madurez de los procesos. Caso: pequeña empresa manufacturera", DYNA Engineering and Industry, 85 (5), DYNA, Bilbao, pp. 392-400 (2010); "Innovación en las Pymes: desarrollo de un modelo, para el estado de Hidalgo”, en José Sánchez (comp.), La competitividad como estrategia en época de crisis, Universidad de Guadalajara, Guadalajara, pp. 211-228 (2010); "Plan estratégico para detonar el desarrollo económico social del municipio de Acaxochitlán”, Innova, 2, Cocytech, Pachuca, pp. 16-19 (2009). 